\title{
EXPERIENCIA INICIAL CON MACOP-B EN LINFOMAS NO-HODGKIN (LNH) DE GRADO INTERMEDIO Y ALTO
}

\author{
Dr. Fernando Checa R. (1), Patricio Gavilanez (2), \\ Dr. Max Ontaneda (2), Dr. Hernán Páez (2).
}

\section{RESUMEN}

En la reunión de la Sociedad Americana de Oncología Clínica de 1986 se confirmaron los resultados de que el esquema MACOP-B (Metotrexate con rescate de ácido folínico, doxorubicina, ciclofosfamida, vincristina, prednisona y bleomicina) era una combinación muy efectiva y tolerada en el tratamiento de LNH de grado intermedio y alto.

Desde mayo de 1986 a noviembre del 87, diez casos avanzados de LNH fueron tratados con MACOP-B, en el Servicio de Oncología del Andrade Marín. Tres fueron mujeres y la edad media de todo el grupo fue 39 años (27-66).. Ocho pacientes presentaron un índice de rendimiento (Karnofsky) de $70 \%$ o más.

De los diez casos, 4 fueron linfoma linfocítico difuso pobremente diferenciado, 2 fueron linfomas indiferencia- dos tipo no Burkitt, 2 fueron linfomas inmunoblásticos y un caso de linfoma histiocítico difuso y linfoblástico, respectivamente.

Se registró 1 paciente en estado II, 4 en estado III y 5 en estado IV.

Todos los pacientes fueron evaluables para toxicidad, siendo la más importante la hematológica. 6 pacientes desarrollaron neutropenia grado 2 (1). Mucositis moderada e intensa se vio en 6. Náusea fue universal, pero vómito grado 2 se registró en 6 pacientes. No se observaron muertes por este esquema. La respuesta completa fue del $70 \%$ y 8 de los 10 pacientes se encuentran vivos con seguimiento mayor a 12 meses.

Nuestra conclusión es que MACOP-B es más efectivo que CHOP o CHOP. Bleo, usados en el servicio hasta mayo 
del 86 , pero tiene una alta toxicidad; por otro lado el costo de tratamiento es muy inferior a otros similares de tercera generación, aunque el costo es mayor que CHOP o CHOP Bleo.

Pensamos seguir utilizando estos esquemas como tratamientos de base para linfomas de grado intermedio y alto, en especial si son pacientes adultos.

\section{SUMMARY}

During the ASCO meeting of 1986 some papers showed that MACOP-B (Metotrexate plus folinic acid rescue, doxorubicin, cyclophosphamide, vincristine, prednisone and bleomycin) was a very effective and well tolerated combination for the treatment of intermediate and high grade Non-Hodgkin Lymphoma.

Ten patients with advance NHL were treated with MACOP-B from may 86 to november 87 in the Oncology Unit of the Carlos Andrade Marin Hospital.

The median age of our patients was 39 years (27-66), and three were females. Eight patients had a performance status of $70 \%$ according to the Karnofsky scale. The following hitological types were found: 4 were poorly differentiated lymphocytic lymphomas, 2 were Inmunoblastic lymphomas, 2 were Undifferentiated lymphomas, 1 was a Diffuse Histiocytic Imphoma and 1 was a Lymphoblastic lymphoma.

There was 1 stage II, 4 stage III and 5 stage IV.

Mielotoxicity was the main side effect with 6 patients developing grade II neutropenia (1). Moderate to intense mucositis was seen in 5 patients. Nau- sea was present in all patients but gra. de II vomiting was registered in $6 \mathrm{pa}$ tients. We have no deaths related to therapy.

Complete response was seen in 7 patients and 8 are alive with a follow up of more than 12 months.

We think that MACOP-B is more effective therapy than CHOP or CHOP-B which was our standar treatment until may-86, although it has a high toxicity rate. It happens to be one of the cheapest among the third generation regimens.

We intend to continue treating patients with this type of chemotherapy.

\section{INTRODUCCION}

En los LNH se obtenían remisiones parciales y muy rara vez se veía una sobrevida prolongada con el uso de agentes quimioterápicos únicos. En un estudio restrospectivo hecho por Jones (2) reportó una respuesta completa (RC) del 5\% en pacientes con Linfoma Histiocítico Difuso (LHD) con una sobrevida media de apenas 7.5 meses.

En los primeros años de la década de los 70 se comenzó a tratar los linfomas difusos de células grandes con esquemas combinados como el MOPP (mostaza nitrogenada, vincristina, procarbazina y prednisona), C-MOPP donde ciclofosfamida reemplaza a la mostaza. De Vita (3) publicó una respuesta completa del $41 \%$ con una sobrevida libre de enfermedad (SLE) del $37 \%$ a los 5 años.

La documentación de que la Doxorubicina (ADR) y blenoxane (BLX) eran 
activos en esta enfermedad llevó a la estructuración de regímenes como el CHOP (4), que contiene ciclofosfamida (CYC), adriamicina (ADR), vincristina (VCR) y prednisona; el BACOP (5) y CHOP-Bleo (6) que contienen BLX a más de las drogas anteriores. Respuestas completas del $60 \%$ o más han sido reportadas con el uso de estas combinaciones, aunque había un grupo substancial de recurrencia (40\%), incluso con quimioterapia (QT) de mantenimiento. Seguimiento de pacientes tratados con CHOP-Bleo en una sola institución (7), mostraron una RC del $65 \%$ y una SLE a los 10 años del $69 \%$ para los pacientes que tuvieron $\mathrm{RC}$.

A finales de la década de los 70 varios estudios demostraron la actividad de varios antimetabólicos como el Metotrexate (MTX), citarabina (CTB) contra los linfomas. Varios esquemas como el COMLA (8), M-BACOP (9), y ProMACE-MOPP (10) fueron estudiados. Estos esquemas usan agentes mielo y nomielotóxicos, alternando en forma secuencial con regímenes que no tengan resistencia cruzada. Con Pro-MACEMOPP se obtuvo remisiones completas del $74 \%$ y SLE del $61 \%$ a los tres años.

Gracias a todos estos ensayos clínicos se pudo establecer que la QT podía lograr RC de $41 \%$ a $77 \%$ con SLE de $35 \%$ a $60 \%$.

Con la introducción definitiva de drogas no-mielosupresoras como VCR, BLX y MTX con rescate de leucovorin (RLE) se elaboraron esquemas de administración en tiempos más cortos.

Uno de estos esquemas es el MACOP-B (11) (MTX+RLE,ADR,CYC,VCR,
BLX y prednisona), el que tiene ciertas características: se administra en un tiempo de 12 semanas, administración intravenosa de agentes mielo y no-mielosupresores en semanas alternas, se indicaprednisona y co-trimoxazole durante todo el tratamiento y se usa dosis moderadamente altas de MTX con RLE.

La experiencia del servicio de Ontolocía del Hospital CAM de Quito en los primeros 10 pacientes con LNH difusos, tratados con el esquema MACOP-B es el motivo del presente estudio.

\section{MATERIAL Y METODOS}

Entre mayo de 1986 y noviembre del 87, 10 casos con estadios avanzados de grado intermedio y alto de LNH fueron tratados con el esquema MACOP-B.

Los pacientes fueron completamente evaluados tanto clínica como radiológicamente, incluyendo TAC de abdomen. Todos los casos tuvieron comprobación histológica y esta fue revisada por uno de nuestros patólogos. La clasificación por estadios clínicos utilizada, fue la de Ann Arbor. Estados avanzados se consideró los III y IV, pero incluimos un caso de linfoma gástrico de mediano volumen.

A excepción de un solo paciente que recibió 6 ciclos de COP (CYC, VCR y y PDN) fuera de nuestra institución, todos los demás no habían recibido nıngún tratamiento previo.

Se siguió lo más estrictamente posible el programa de tratamiento delineado en la publicación de Klimo en 1985 (11), aunque tres pacientes sufrieron retrasos de hasta 2 semanas por 
falta de medicamentos, en especial del leucovorin. Sólo uno de nuestros pacientes tuvo compromiso de médula, pero no se administró QT intratecal. Dos pacientes recibieron radioterapia de consolidación: uno sobre estómago y otro sobre todo el abdomen.

\section{Respuesta}

Se consideró respuesta completa la desaparición total de todos los signos y síntomas clínicos presentes antes del tratamiento. La evaluación se hizo a la sexta semana.

Respuesta parcial se consideró cuando no hubo ausencia de enfermedad, pero las lesiones no habían progresado.

La sobrevida se calculó desde el momento que se inició el tratamiento.

\section{RESULTADOS}

Las características de los pacientes se encuentran detalladas en la Tabla No 1 y los resultados de tratamiento en la Tabla № 2.

Siete de los pacientes tratados tuvieron una $\mathrm{RC}(70 \%)$, con 2 pacientes con RP, traduciéndose por lo tanto en un $90 \%$ la respuesta total. En 5 de los 8 pacientes que se encuentran vivos sin enfermedad la respuesta se produjo dentro de las primeras 4 semanas de tratamiento. En un paciente se documentó mejoría casi completa con gastrocopía a la 6ta. semana. Los 8 pacientes vivos tienen un seguimiento mínimo de 6 meses y máximo de 21 meses.

Los fallecimientos ocurrieron luego de 4 y 16 meses de postramiento; am- bos por recurrencia de enfermedad Uno de ellos fue sometido a QT de rescate con buena respuesta inicial, pero al 3er. ciclo abandonó el tratamiento.

En lo que se refiere a toxicidad todos los pacientes desarrollaron alopecia. La toxicidad más importante fue la hematológica con dos pacientes, requiriendo hospitalización por granulocitopenia $(500 / \mathrm{mm} 3)$. Granulocitopenia moderada se vio en 6 pacientes por una o dos veces durante tratamiento.

Mucositis oral severa (algunos pacientes se vieron impedidos de alimentarse por varios días) se observó en 3 pacientes y moderada en otros 3 .

Náusea y vómito moderado se desarrolló en 5 pacientes. Otros eventos complicaron el curso del tratamiento como tromboflebitis, acné, herpes zoster, neuritis periférica, en una ocasión cada uno.

No hemos tenido casos de toxicidad pulmonar o cardíaca en nuestra serie.

\section{DISCUSION}

El advenimiento de nuevos esquemas de QT combinada ha producido un cambio radical en el pronóstico de aquellos linfomas conocidos por su mal pronóstico. El alto porcentaje de respuestas completas $(80 \%)$ con una relativa baja en la frecuencia de recidivas, han producido sobrevidas de 3 años superiores al $70 \% 80 \%$.

En una reciente revisión hecha por Connors en 1986 (11) indica una recidiva del $16 \%$ y una SLE global del $66 \%$ para todos los pacientes. 
Las RC de nuestros pacientes muestran una alta probabilidad de que conforme se reúnan más pacientes la proporción se mantendrá alrededor del $80 \%$, con un bajo porcentaje.

En vista de que este tratamiento se empezó a utilizarse en mayo del 85 tendremos que dejar transcurrir más tiempo para ver cómo se comportan nuestros pacientes, sin embargo por reportes de Klimo, en donde ningún paciente hasta el momento ha rrecurrido luego de 12 meses, esperamos que los casos nuestros que han sobrepasado este tiempo (4/8), evolucionen sin recidivas. Es interesante anotar que uno de nuestros pacientes recurrió a los 16 meses, luego de lo cual falleció pese a intentar rescate.

Lo que más nos preocupa de este esquema es su toxicidad, en especial la granulocitopenia marcada, que necesita estrecha vigilancia y la mucositis oral que impide la alimentación de los pacientes y fácilmente les obliga a perder peso.

La introducción de co-trimoxazole y de ketoconazole mantiene el riesgo de infecciones a un nivel mínimo.

Creemos que las otras variables como edad, sexo, histología, estadio clínico reflejan una población heterogénea semejante a la de grupos reportados por otros autores que presentan un número corto de casos.

Por último desearíamos añadir que en una mesa redonda realizada en Indiana (13), se analizó el costo de varios esquemas de tercera generación y el costo del MACOP-B fue el más bajo, cosa de importancia para el tratamiento de nuestros pacientes que no cuentan con grandes recursos económicos. 
TABLA No 1

CARACTERISTICAS CLINICAS DE 10 PACIENTES TRATADOS CON MACOP-B

Pacientes

Hombres/Mujeres

Síntomas

$$
\mathrm{A} / \mathrm{B}
$$

Edad

Promedio/Rango

Karnofsky

$70 \%$

Histología

LLD.PD.

L. Inmunoblástico

L. Indif. No-Burkitt

LHD

L. Linfoblástico

Estadio

II

III

IV

Compromiso extranodal

Abdomen + GI

Pulmón, piel, hueso, órbita, médula
$8 / 2$

$4 / 6$

$39.9 / 27-66$

9

4

2

2

1

1

\section{1}

4

5

7

$1 \mathrm{c} / \mathrm{u}$.

TABLA $N^{\circ} 2$

RESPUESTA A TRATAMIENTO MACOP-BLEO, 10 PACIENTES

\begin{tabular}{lcccc}
\hline & Total & RC & RP & PR \\
\hline LLD.PD. & 4 & 3 & 1 & - \\
L. Inmunobl. & 2 & 1 & 1 & - \\
L. Indifer. & 2 & 2 & - & - \\
LHD & 1 & 1 & - & - \\
L. Linfobl. & 1 & - & - & 1 \\
\hline Estado II & 1 & 1 & - & - \\
III & $4^{4}$ & $3^{3}$ & - & 1
\end{tabular}




\section{BIBLIOGRAFIA}

1.- Miller, A. B., Hoogstraten, B., et al: Cancer, 1981; 47:207.

2.- S. E. Rosenberg, et al.: Non-Hodgkin Lymphomas. II Single Agent Chamotherapy. Cancer, 30:31-38, 1972.

3.- De Vita, V. T., Canellos, G. et al: Advance Histiocytic Lymphoma: A potencially curable disease. Lancet I (7901): 248-250, 1975.

4.- Jones, S. E., Grozea, P. N.: Al Superiority of adriamycin containing combination chemotherapy in the treatment of diffuse lymphomas Cancer, 43:417-425, 1979.

5.- Skarin, A. T., Rosenthal, D. S. et al: Combination chemotherapy of advance nonHodgkin limphomas with bleomycin, adriamycin, ciclophosphamide, vincristine and prednisone (BACOP), Blood 49:757-770, 1977.

6.- Rodríguez, V., Cabanillas, F. et al: Combination chemotherapy (CHOP-BLEO) in advanced (non-Hodgkin) malignant lymphoma. Blood, 49:325-333, 1977.

7.- Lee, R., Cabanillas, F. et al: A 10 year Update of CHOP-BLEO in the treatment of difusse Large Cell Lymphoma.
J. C. Oncology, 4:1455-1461, 1986.

8.- Sweet, D. L., Golomb, H. M. et al: COMLA conbination seguential chemotherapy for advanced diffuse histiocytic lymphoma. Ann Inter Med, 92:785-790, 1980.

9.- Skarin, A. T., Canellos, G. P. et al: Improved prognosis in diffuse histiocytic and undifferentiated by use of hihg dose MTX alternating with standar agents (M-BACOD), J. Clin - Oncl., 1:91-97, 1983.

10.- Fisher, R. I., De Vita, V. T. et al: Diffus agressive lymphomas: Increased survival after alternating flexible sequences of Pro-MACE and MOPP chemotherapy. Ann Int Med, 98:304-309, 1983.

11.- Klimo, P., Connors, J.: MACOP-B chemotherapy for the treatment of Diffuse Large Cell Lymphoma. Ann Int Medic, 102: 596-602, 1985.

12.- Winoko, S. H., Canellos, G.: Current Concepts in the Mangement of Non-Hodgkin Lymphomas. A Round Table Discution. Bristol Myers, Indiana, 1987. 